Rolf Spirig • Elisabetta Peduzzi • Manuel E. Patarroyo • Gerd Pluschke · Claudia A. Daubenberger

\title{
Structural and functional characterisation of the Toll like receptor 9 of Aotus nancymaae, a non-human primate model for malaria vaccine development
}

Received: 13 December 2004 / Revised: 8 February 2005 / Published online: 8 April 2005

C) Springer-Verlag 2005

\begin{abstract}
In the absence of suitable rodent animal models for Plasmodium falciparum malaria, the efficacy testing of asexual blood-stage vaccine candidates in Aotus nancymaae represents a tool to select between different formulations before conducting expensive human clinical trials. $\mathrm{CpG}$ oligonucleotides (ODN) specifically promote the production of pro-inflammatory and Th1-type cytokines and they enhance the immunogenicity of co-administered antigens. Toll like receptor 9 (TLR-9) binds directly and sequence-specifically to single-stranded un-methylated CpG-DNA mediating the biological effects of CpG ODN. We cloned and functionally characterised the TLR-9 cDNA of $A$. nancymaae. The cDNA encompassed 3,099 bp predicted to code for 1,032 amino acid residues. Results of homology searches to human TLR-9 suggested that the receptor is 93 and $94 \%$ identical at the nucleotide and amino acid sequence levels, respectively. Stimulation of splenocytes of $A$. nancymaae with $\mathrm{CpG}$ ODN resulted in proliferative responses in all animals analysed. FACS analysis of cultures incubated with $\mathrm{CpG}$ ODN 2006 indicated that the $\mathrm{B}$ cell marker CD20 was up-regulated consistent with B cell activation. The high level of sequence conservation of Aona-TLR-9 reinforces the suitability of $A$. nancymaae as animal model for malaria subunit vaccine development.
\end{abstract}

The nucleotide sequence has been submitted to the GenBank nucleotide sequence database under the accession number AY788894.

R. Spirig · E. Peduzzi · G. Pluschke · C. A. Daubenberger $(\bowtie)$ Department of Medical Parasitology and Biology of Infection, Molecular Immunology, Swiss Tropical Institute,

Socinstrasse 57 ,

4002 Basel, Switzerland

e-mail: Claudia.Daubenberger@unibas.ch

Tel.: +41-61-2848217

Fax: $+41-61-2718654$

M. E. Patarroyo

Fundación Instituto de Inmunología de Colombia,

FIDIC,

Calle 26 No 50-00,

Santa Fe de Bogotá, Bogota, Colombia
Keywords Toll like receptor 9 - Plasmodium falciparum . Aotus nancymaae $\cdot \mathrm{CpG}$ oligonucleotides $\cdot \mathrm{CD} 20$

The vertebrate innate immune system recognises conserved, pathogen-associated molecular patterns and responds through the secretion and activation of a number of effector molecules (Klinman 2004). Recognition of un-methylated CpG di-nucleotides within specific flanking bases has evolved as a pattern recognition mechanism used by the innate immune system to detect the DNA of pathogens (Klinman 2004). The central involvement of the Toll like receptor 9 (TLR-9) in responses to un-methylated $\mathrm{CpG}$ di-nucleotides has been demonstrated using TLR-9-deficient mice (Hemmi et al. 2000). CpG DNA is bound directly by the TLR-9 in the lysosomal compartment after cellular uptake (Cornelie et al. 2004; Latz et al. 2004; Rutz et al. 2004). B cells and plasmacytoid dendritic cells are the main human cell types that express TLR-9 and respond directly to CpG ODN stimulation. Human memory B cells constitutively express high levels of TLR-9 and proliferate and differentiate to immunoglobulin-secreting cells in response to $\mathrm{CpG}$ (Bernasconi et al. 2003). CpG ODN interact with TLR-9 expressed by plasmacytoid dendritic cells, improving antigen presentation and triggering the production of chemokines, Th1-type and pro-inflammatory cytokines (including INF- $\gamma$, IL-6, IL12 , IL-18 and TNF- $\alpha$ ). Hence, CpG ODN have great potential to be developed as adjuvant for T- and B-cell responses (Klinman 2004).

At least three structurally distinct classes of CpG ODN have been described in primates. K-type CpG ODN induce strong B- and NK-cell activation, D-type ODN provoke high levels of IFN- $\alpha$ secretion by plasmacytoid dendritic cells, whilst C-type ODN combine properties of K- and D-type ODN (Vollmer et al. 2004). Examples of K-type include ODN 2006, an ODN with four "TCGT" motifs separated by poly-T (Hartmann et al. 2000). Addition of $\mathrm{K}$-type ODN to vaccines against influenza virus, measles virus, hepatitis $\mathrm{B}$ virus surface antigen or tetanus toxoid increased antigen-specific antibody titres in mice by up to 
Fig. 1 a Amino acid sequence comparison of TLR-9 of $A$. nancymaae and $H$. sapiens using the ClustalW program available at http://www.ebi.ac. uk/clustalw/. Symbols “*”, “:”" and "." indicate identical, conserved and semi-conserved amino acid residues, respectively. Both sequences were analysed for their domain structure using the SMART program available at http://www.smart. embl-heidelberg.de/. The predicted signal sequence is given in italics, the leucine-rich repeat region $(L R R)$ is underlined and the boxed sequences indicate the Toll-interleukin 1-resistance domain $(T I R)$. b Unrooted phylogenetic tree established from deduced TLR-9 amino acid sequences of Canis familiaris (NP_001002998), Sus scrofa (NP-999123), Rattus norvegicus (NP 937764), Homo sapiens (NP 059138), Felis catus (AAN15751), Mus musculus (NP 112455) and A. nancymaae AY788894 using the neighbour-joining algorithm of the PHYLIP 3.572 program package available at http://www. bioweb.pasteur.fr. The numbers at the nodes indicate the percentage of recovery of that node in 1,000 bootstrap replications a

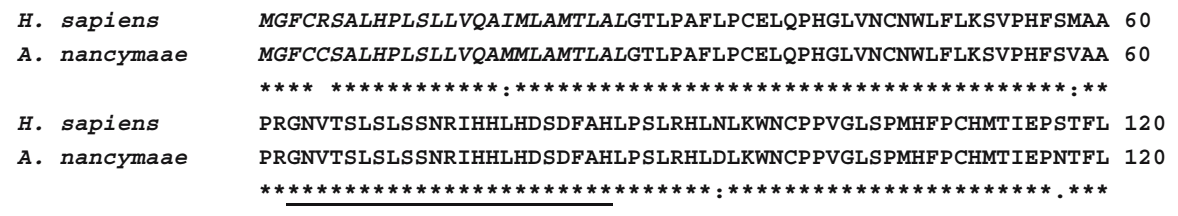

H. sapiens AVPTLEELNLSYNNIMTVPALPKSLISLSLSHTNILMLDSASLAGLHALRFLFMDGNCYY 180 A. nancymaae AVPTLEELNLSYNSITAVPALPKSLVSLTLSRTNILVLDSASLAGLHALRFLFMDGNCYY 180

H. sapiens

A. nancymaae

H. sapiens

A. nancymaae

H. sapiens

A. nancymaae

H. sapiens

A. nancymaae

H. sapiens

A. nancymaae

H. sapiens

A. nancymaae

H. sapiens

A. nancymaae

H. sapiens

A. nancymaae

H. sapiens

A. nancymaae

H. sapiens

A. nancymaae

H. sapiens

A. nancymaae

H. sapiens

A. nancymaae

H. sapiens

A. nancymaae

H. sapiens

A. nancymaae

H. sapiens

A. nancymaae

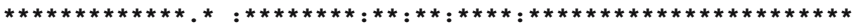

KNPCRQALEVAPGALLGLGNLTHLSLKYNNLTVVPRNLPSSLEYLLLSYNRIVKLAPEDL 240 KNPCRRALEVAPGALLGLGNLTHLSLKYNNLTVVPRNLPSSLEYLLLSYNHIIKLAPGDL 240

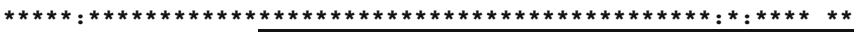

ANLTALRVLDVGGNCRRCDHAPNPCMECPRHFPQLHPDTFSHLSRLEGLVLKDSSLSWLN 300 ANLTALRVLDVGGNCRRCDHAPNPCMECPRHFPQLHPNTFSHLSRLEGLVLKDSSLSWLN 300 ****************************************:***********************

ASWFRGLGNLRVLDLSENFLYKCITKTKAFQGLTQLRKLNLSFNYQKRVSFAHLSLAPSF 360 ASWFHGLGNLRVLDLSENFLYECITKTKAFQGLTQLRKLNLSFNYQKRVSFAHLSLAPSF 360

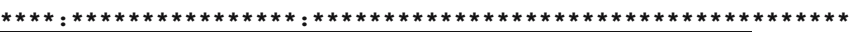

GSLVALKELDMHGIFFRSLDETTLRPLARLPMLQTLRLQMNFINQAQLGIFRAFPGLRYV 420 GSLFSLEELDMHGIFFRSLDETTLRPLARLSRLQTLHLQMNF INQAQLGIFGAFPGLRHV 420

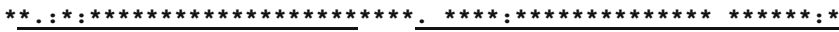

DLSDNRISGASELTATMGEADGGEKVWLQPGDLAPAPVDTPSSEDFRPNCSTLNFTLDLS 480 DLSDNRISGASELAATTGEADGGGRVWLQPGDLAPAPVDAPSSEDFTANCSTLNFTLDLS 480 $* * * * * * * * * * * * *: * * * * * * * *:: * * * * * * * * * * * * * *: * * * * * * \quad . * * * * * * * * * * * *$

RNNLVTVQPEMFAQLSHLQCLRLSHNCISQAVNGSQFLPLTGLQVLDLSHNKLDLYHEHS 540 RNNLVTVRPEMFARLSHLQCLRLSHNCIMQAVNGSQFLPLTDLRVLDLSHNKLDLYHEHS 540 $* * * * * * *: * * * * *: * * * * * * * * * * * * * * * * * * * * * * * * * * * * . *: * * * * * * * * * * * * * * * * *$

FTELPRLEALDLSYNSQPFGMQGVGHNFSFVAHLRTLRHLSLAHNNIHSQVSQQLCSTSL 600 FTELPRLEALDLSYNSQPFGMQGVGHNFSFVAHLRTLRYLSLAHNNIHSRVSPQLRSTSL 600 $* * * * * * * * * * * * * * * * * * * * * * * * * * * * * * * * * * * * * * *: * * * * * * * * * * *: * * * * * * * * *$

RALDFSGNALGHMWAEGDLYLHFFQGLSGLIWLDLSQNRLHTLLPQTLRNLPKSLQVLRL 660 RALDFSGNALGRMWAEGDLYLHFFQGLSGLIWLDLSQNSLHTLLPRTLGNLPKTLQVLRL 660 $* * * * * * * * * * *: * * * * * * * * * * * * * * * * * * * * * * * * * * * * * * * * * *: * * * * * *: * * * * * *$

RDNYLAFFKWWSLHFLPKLEVLDLAGNQLKALTNGSLPAGTRLRRLDVSCNSISFVAPGF 720 RDNKLAFFKWRSLALLRKLEALDLAGNQLKALTNGSLPAMTRLRKLDVSCNSISFVAPGF 720 $* * * * * * * * * * *:: * * * * . * * * * * * * * * * * * * * * * * * * * * * * * * * * * * * * * * * * * *$ FSKAKELRELNLSANALKTVDHSWFGPLASALQILDVSANPLHCACGAAFMDFLLEVQAA 780 FSKAKKLRELNLSANALKTVDPSWFGPLVGALKILDVSTNPLHCACGAAFVDFLLEVQAA 780 $\star * * * *: * * * * * * * * * * * * * * * * * * * * * * \ldots * *: * * * * *: * * * * * * * * * * * *: * * * * * * * * *$

VPGLPSRVKCGSPGQLQGLSIFAQDLRLCLDEALSWDCFALSLLAVALGLGVPMLHHLCG 840 VPGLPSRVRCGSPAQLQGQSIFAQDLRLCLDEALSRDCFSFSLLAVALGLGVPLLHQLCG 840 $* * * * * * * *: * * * * . * * * * * * * * * * * * * * * * * * * * * * *:: * * * * * * * * * * * *: * *: * * * *$ WDLWYCFHLCLAWLPWRGRQSGRDEDAL PYDAFVVFDKTQSAVADWVYNELRGQLEECRG 900 WDLWYCFYLGLAWLPRWGRRGGRREDAL PYDAFVVFDKAQSAVADWVYNELRRQLEECRG 900 $* * * * * * *: * * * * * * \quad * *: . * * * * * * * * * * * * * * * *: * * * * * * * * * * * * * * * * * * * *$ RWALRLCLEERDWLPGKTLFENLWASVYGSRKTLFVLAHTDRVSGLLRASFLLAQQRLLE 960 RWALRLCLEERDWLPGKTLFENLWASVYGSRKTLFVLAHTDRVSGLLRASFLLAQQQRLLE 960 $* * * * * * * * * * * * * * * * * * * * * * * * * * * * * * * * * * * * * * * * * * * * * * * * * * * * * * * * * * * * * *$ DRKDVVVLVILSPDGRRSRYVRLRQRLCRQSVLLWPHQPSGQRSFWAQLGMALTRDNHHF 1020 DRKDVVVLVILSPEGCRSRYVRLRQRLCRQSVLLWPHQPSGQRSFWAQLGIALTGDNHHF 1020 $* * * * * * * * * * * * *: * * * * * * * * * * * * * * * * * * * * * * * * * * * * * * * * * * * *: * * * * * * * *$ YNRNFCQGPTAE 1032 YNRNFCQGPTAE 1032 $* * * * * * * * * * * *$
3 orders of magnitude (Klinman 2004). Optimal immunostimulatory CpG DNA motifs differ between mouse and human based on amino acid sequence variations between the extracellular regions of TLR-9 (Bauer et al. 2001). Therefore, experiments to determine whether a particular CpG ODN may be of therapeutic benefit in humans are initiated in rodents, followed by evaluations in non-human primates finally leading to phase I clinical trials (Cooper et al. 2004; Krieg et al. 2004).
The development of a safe and effective malaria vaccine remains an urgent medical need for human populations living in malaria-endemic regions (Malaney et al. 2004; Richie and Saul 2002). Aotus nancymaae is one of the few permissive hosts for the asexual forms of two major human malaria parasites, Plasmodium falciparum and P. vivax (Gysin 1998). Immunisation and parasite challenge studies in $A$. nancymaae can rationally support decisions concerning further development of subunit malaria vaccines based on 
Fig. 1 (continued)

b

Felis catus

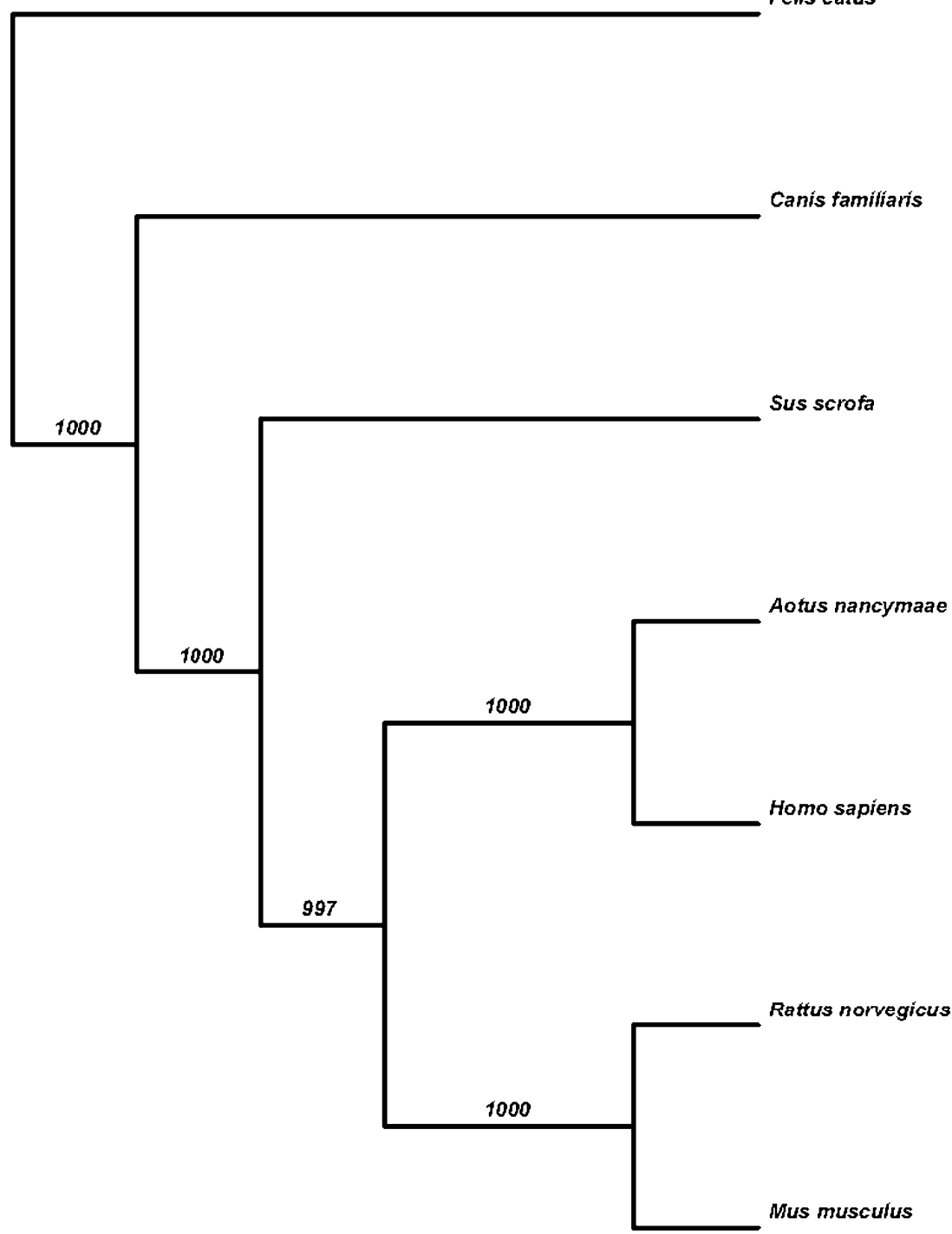

distinct antigens, antigen combinations and antigen delivery systems (Chang et al. 1996). Aluminium compounds, the only adjuvant used widely in humans, do not support the development of Th1-type cell-mediated immune responses. Since this arm of the cellular immune response seems to play a central role in protection against a number of infectious diseases including malaria (Gupta 1998), novel approaches to develop vaccine formulations that induce Th1-type and cytotoxic $\mathrm{T}$ cell responses are needed.

We carried out immunogenetic studies to characterise the adaptive immune system of $A$. nancymaae in order to define at the molecular level its suitability as an experimental model in asexual blood-stage malaria vaccine development (Daubenberger et al. 2001; Diaz et al. 2000a,b, 2002; Favre et al. 1998; Nino-Vasquez et al. 2000; Vecino et al. 1999). In the current study, we characterised the cDNA sequence of the TLR-9 of $A$. nancymaae (AonaTLR-9), followed by the description of in vitro stimulation of A. nancymaae spleen cells by distinct CpG ODN. This study was motivated by the hypothesis that $\mathrm{CpG}$ ODN might have suitable adjuvant activity for the induction of Th1-mediated immune responses in malaria vaccine development (Jones et al. 1999). Additionally, a recent report demonstrated that schizont extracts of $P$. falciparum may contain a novel non-CpG ODN ligand binding to human TLR-9. Investigations of this novel TLR-9 malaria parasite interaction in a suitable animal model may lead to better insights into early events in host-parasite interactions in malaria (Pichyangkul et al. 2004).

The analysed animals were captured in the Colombian Amazon area close to Leticia and kept at the experimental primate station of the Fundacion Instituto de Immunologia de Colombia (FIDIC) in accordance with the recommendations of the Committee on Care and Use of Laboratory Animals, US and the Colombian National Institute of Health guidelines for use of laboratory animals and supervised by the Colombian Wildlife Corporation (CORPOAMAZONIA). Mononuclear cells from three healthy monkeys numbered as 23964, 23967 and 23968 were obtained by splenectomy followed by density gradient separation. Total RNA was 
Fig. 2 Proliferative responses of spleen cells of $A$. nancymaae to CpG ODN 2006. A. nancymaae spleen cells of three animals were cultured in the absence or presence of $6 \mu \mathrm{g} / \mathrm{ml} \mathrm{CpG} \mathrm{ODN}$ for $72 \mathrm{~h}$, pulsed during the last $16 \mathrm{~h}$ with $\left[{ }^{3} \mathrm{H}\right]$ thymidine and incorporated radioactivity was measured. Data represent mean counts per minute $(\mathrm{cpm})$ of triplicate cultures and the standard error of means of triplicate cultures was always $<15 \%$

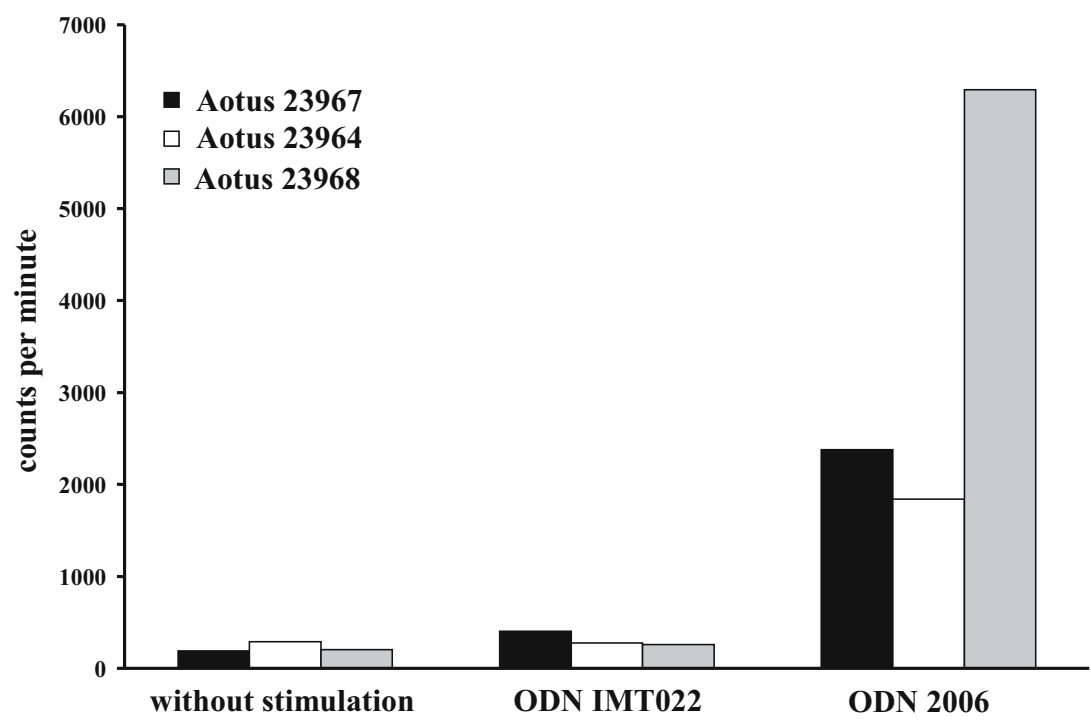

isolated from spleen cells of monkey 23968 and cDNA synthesised as described (Daubenberger et al. 2001). Using primer pairs designed according to the human TLR-9 sequence (GenBank accession number NM017442) and located either outside (N- and C-terminal ends) or inside the coding sequence, a set of overlapping RT-PCR products were obtained which comprised the entire coding sequence of $A$. nancymaae TLR-9 (Aona-TLR-9) cDNA. To exclude possible PCR errors, all products were amplified from two independently obtained cDNA, and at least four independent plasmid preparations of each PCR amplification reaction were sequenced. The total length of the Aona-TLR-9 open reading frame (ORF) was 3,099 bp coding for a predicted protein of 1,032 amino acids. The nucleotide and the amino acid sequences displayed 93 and 94\% identity, respectively, to the human TLR-9. Alignment of the deduced amino acid sequences of Aona-TLR-9 and human TLR-9 is shown in Fig. 1a. A comparison of structural features using the SMART architecture research programme http://www. smart.embl-heidelberg.de) demonstrated that the structure of Aona-TLR-9 is highly conserved to human TLR-9. Eighteen leucine-rich repeats (LRR) were identified by the programme, followed by one leucine-rich repeat C-terminal domain and the toll interleukin 1-resistance domain (TIR) encompassing residues 869 to 1,016 (Fig. 1a). A singlenucleotide polymorphism of TLR-9 in human populations has been described (Lazarus et al. 2003). One of the allelic variants in humans carried at amino acid position 5 a cysteine instead of arginine (R5C). Alignment of human and Aotus TLR-9 amino acid sequences demonstrated the same sequence polymorphism (R5C) between these two species (Fig. 1a). TLR-9 is a type I membrane receptor displaying an N-terminal extracellular region abundant in leucine-rich repeats, a cysteine-rich domain to the membrane, a single transmembrane region and a cytoplasmic region comprising the conserved TIR domain. A potential CpG-DNA binding domain of TLR-9 located between amino acid residues 523 and 541 has been defined (Rutz et al. 2004). Introduction of double alanine mutations (D535A and Y537A) strongly diminished the CpG-sequence-specific DNA binding, supporting the notion that the region containing D535 and Y537 is involved in DNA binding. These two amino acid residues are conserved between all full-length TLR-9 sequences available in the GeneBank including Aona-TLR-9.

Then we conducted a phylogenetic analysis incorporating all full-length TLR-9 sequences available at GenBank. The analysis was performed employing the PHYLIP 3.572 software package available at http://www.bioweb.pasteur.fr and the phylogenetic tree was constructed according to the neighbour-joining method based on Kimura two-parameter distances estimates (Saitou and Nei 1987). The results of this analysis, shown in Fig. 1b, confirm that the TLR-9 of human and Aotus are closely related, clustering together on a separate branch and separated from rodent and carnivora TLR-9 sequences.

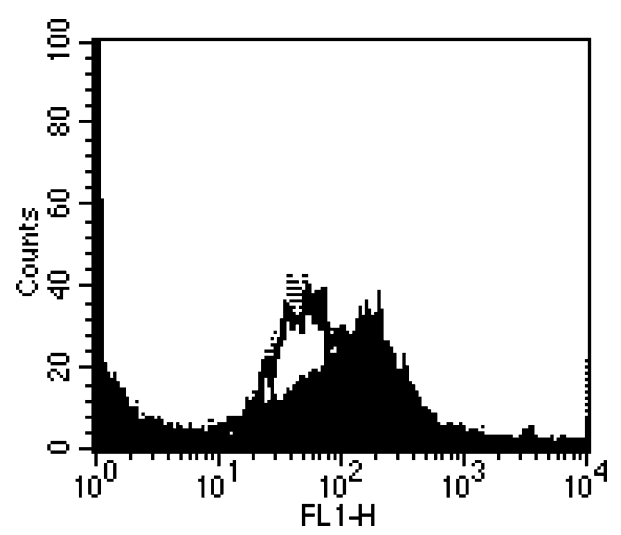

Fig. 3 FACS analysis of $A$. nancymaae splenocytes after CpG ODN incubation. Spleen cells were incubated for $96 \mathrm{~h}$ with medium alone (dotted line), control CpG ODN IMT022 (black line) and ODN 2006 (filled graph) at $6 \mu \mathrm{g} / \mathrm{ml}$. Cells were removed from culture and stained with anti-CD20 mAb B-Ly1 followed by incubation with FITClabelled anti-mouse IgG antibodies and analysed by FACS analysis 
Next, we wanted to demonstrate that $\mathrm{CpG}$ ODN activate the spleen cells of $A$. nancymaae. It is well established that $\mathrm{K}$-type CpG ODN induce proliferative responses via TLR-9 binding in B cells (Krieg et al. 1995). Therefore, we measured the proliferative responses of splenocytes upon incubation with ODN 2006 and control ODN IMT022. The K-type CpG ODN 2006 was chosen because of its previously described stimulatory activities in cells derived from human, chimpanzee and rhesus monkey (Hartmann et al. 2000). As control, we included ODN IMT022 that has also been described previously (Elias et al. 2003). Desalted phosphoro-thioate oligonucleotides (ODN), HPLC-purified, were purchased from Metabion $\mathrm{GmbH}$ (Germany). Purity was assessed by HPLC assays. ODN were suspended in HBSS buffer (Sigma) and kept at $-20^{\circ} \mathrm{C}$ until use. $A$. nancymaee spleen cells $\left(1 \times 10^{5}\right.$ cells/well) were stimulated for $72 \mathrm{~h}$ with ODN 2006 and control ODN IMT022 at $6 \mu \mathrm{g} / \mathrm{ml}$ in 96-well round-bottomed plates or with medium alone. Sixteen hours before harvest, $\left[{ }^{3} \mathrm{H}\right]$ thymidine was added $(1 \mu \mathrm{Ci} /$ well $)$ into the cultures and the incorporated radioactivity measured by scintillation counting. In cultures of splenocytes of all three Aotus animals tested, ODN 2006 induced significant proliferative responses. The $\left[{ }^{3} \mathrm{H}\right]$ thymidine uptake in cultures incubated with control ODN IMT022 was comparable to un-stimulated cultures, demonstrating that the effect of ODN 2006 was dependent on a distinct nucleotide sequence (Fig. 2).

In order to confirm that Aotus B cells were activated by CpG ODN 2006, we analysed the changes in CD20 expression level. The CD20 molecule, also known as Bp35, is expressed from the pre-B to mature B cell stages, and whilst its precise functional role remains unknown, a role in B cell activation, proliferation and differentiation is suggested (Clark et al. 1985). Briefly, spleen cells of A. nancymaae were cultivated at $3 \times 10^{5}$ cells $/ \mathrm{ml}$ in cell culture medium in 48-well plates (Nunc) for $96 \mathrm{~h}$ in the presence of medium alone or ODN 2006 or IMT022. Cells were recovered, transferred to FACS tubes and stained for flow cytometry with the anti-CD20 mAb B-Ly1 (Pharmingen) (Daubenberger et al. 2001). Cells were gated using forward and side scatter parameters for dead cell exclusion and in each sample, 10,000 events were measured and data analysed using the CellQuest software (BD Biosciences). As demonstrated in Fig. 3, incubation of spleen cells with ODN 2006 resulted in a shift of the cell population to the right of the FL1-H axis. In cell cultures incubated with medium and control ODN IMT022, the mean fluorescence intensities were 180 and 141, respectively, whilst in ODN 2006 treated cultures a value of 202 could be measured. Provided that mAb B-Ly1 stains the CD20 homologue in A. nancymaae, these results indicate, to our knowledge, for the first time that CD20 might be upregulated upon incubation of splenocytes with CpG ODN 2006.

In summary, the first complete TLR-9 cDNA sequence of a non-human primate is described in this work. Aotus monkeys immunised with a synthetic malaria peptide formulated in CpG ODN 2006 responded with significantly higher antibody levels compared to animals treated with control ODN (Jones et al. 1999). In combination with our mo- lecular and functional characterisation of the Aona-TLR-9, these results support the suitability of Aotus monkeys in the analysis of $\mathrm{CpG}$ ODN as an adjuvant compound for subunit-based vaccines against $P$. falciparum and $P$. vivax.

\section{References}

Bauer S, Kirschning CJ, Hacker H, Redecke V, Hausmann S, Akira S, Wagner H, Lipford GB (2001) Human TLR9 confers responsiveness to bacterial DNA via species-specific CpG motif recognition. Proc Natl Acad Sci U S A 98:9237-9242

Bernasconi NL, Onai N, Lanzavecchia A (2003) A role for Toll-like receptors in acquired immunity: up-regulation of TLR9 by BCR triggering in naive $\mathrm{B}$ cells and constitutive expression in memory B cells. Blood 101:4500-4504

Chang SP, Case SE, Gosnell WL, Hashimoto A, Kramer KJ, Tam LQ, Hashiro CQ, Nikaido CM, Gibson HL, Lee-Ng CT, Barr PJ, Yokota BT, Hut GS (1996) A recombinant baculovirus 42-kilodalton C-terminal fragment of Plasmodium falciparum merozoite surface protein 1 protects Aotus monkeys against malaria. Infect Immun 64:253-261

Clark EA, Shu G, Ledbetter JA (1985) Role of the Bp35 cell surface polypeptide in human B-cell activation. Proc Natl Acad Sci U S A 82:1766-1770

Cooper CL, Davis HL, Morris ML, Efler SM, Krieg AM, Li Y, Laframboise C, Al Adhami MJ, Khaliq Y, Seguin I, Cameron DW (2004) Safety and immunogenicity of CPG 7909 injection as an adjuvant to Fluarix influenza vaccine. Vaccine 22:31363143

Cornelie S, Hoebeke J, Schacht AM, Bertin B, Vicogne J, Capron M, Riveau G (2004) Direct evidence that Toll-like receptor 9 (TLR9) functionally binds plasmid DNA by specific cytosinephosphate-guanine motif recognition. J Biol Chem 279:1512415129

Daubenberger CA, Salomon M, Vecino W, Hubner B, Troll H, Rodriques R, Patarroyo ME, Pluschke G (2001) Functional and structural similarity of $\mathrm{V}$ gamma $9 \mathrm{~V}$ delta $2 \mathrm{~T}$ cells in humans and Aotus monkeys, a primate infection model for Plasmodium falciparum malaria. J Immunol 167:6421-6430

Diaz D, Naegeli M, Rodriguez R, Nino-Vasquez JJ, Moreno A, Patarroyo ME, Pluschke G, Daubenberger CA (2000a) Sequence and diversity of MHC DQA and DQB genes of the owl monkey Aotus nancymaae. Immunogenetics 51:528-537

Diaz OL, Daubenberger CA, Rodriguez R, Naegeli M, Moreno A, Patarroyo ME, Pluschke G (2000b) Immunoglobulin kappa light-chain V, J, and $\mathrm{C}$ gene sequences of the owl monkey Aotus nancymaae. Immunogenetics 51:212-218

Diaz D, Daubenberger CA, Zalac T, Rodriguez R, Patarroyo ME (2002) Sequence and expression of MHC-DPB1 molecules of the New World monkey Aotus nancymaae, a primate model for Plasmodium falciparum. Immunogenetics 54:251-259

Elias F, Flo J, Lopez RA, Zorzopulos J, Montaner A, Rodriguez JM (2003) Strong cytosine-guanosine-independent immunostimulation in humans and other primates by synthetic oligodeoxynucleotides with PyNTTTTGT motifs. J Immunol 171:3697-3704

Favre N, Daubenberger C, Marfurt J, Moreno A, Patarroyo M, Pluschke G (1998) Sequence and diversity of T-cell receptor alpha V, J, and C genes of the owl monkey Aotus nancymaae. Immunogenetics 48:253-259

Gupta RK (1998) Aluminum compounds as vaccine adjuvants. Adv Drug Deliv Rev 32:155-172

Gysin J (1998) Animal models: primates. In: Sherman IW (ed) Malaria: parasite biology, pathogenesis, and protection. ASM Press, Washington DC, pp 419-439

Hartmann G, Weeratna RD, Ballas ZK, Payette P, Blackwell S, Suparto I, Rasmussen WL, Waldschmidt M, Sajuthi D, Purcell RH, Davis HL, Krieg AM (2000) Delineation of a CpG phosphorothioate oligodeoxynucleotide for activating primate immune responses in vitro and in vivo. J Immunol 164:1617-1624 
Hemmi H, Takeuchi O, Kawai T, Kaisho T, Sato S, Sanjo H, Matsumoto M, Hoshino K, Wagner H, Takeda K, Akira S (2000) A Toll-like receptor recognizes bacterial DNA. Nature 408:740745

Jones TR, Obaldia N III, Gramzinski RA, Charoenvit Y, Kolodny N, Kitov S, Davis HL, Krieg AM, Hoffman SL (1999) Synthetic oligo-deoxynucleotides containing $\mathrm{CpG}$ motifs enhance immunogenicity of a peptide malaria vaccine in Aotus monkeys. Vaccine 17:3065-3071

Klinman DM (2004) Immunotherapeutic uses of CpG oligodeoxynucleotides. Nat Rev Immunol 4:249-258

Krieg AM, Yi AK, Matson S, Waldschmidt TJ, Bishop GA, Teasdale R, Koretzky GA, Klinman DM (1995) CpG motifs in bacterial DNA trigger direct B-cell activation. Nature 374:546-549

Krieg AM, Efler SM, Wittpoth M, Al Adhami MJ, Davis HL (2004) Induction of systemic TH1-like immunity in normal volunteers following subcutaneous but not intravenous administration of CPG 7909, a synthetic B-class CpG oligo-nucleotide TLR9 agonist. J Immunother 27:460-471

Latz E, Schoenemeyer A, Visintin A, Fitzgerald KA, Monks BG, Knetter CF, Lien E, Nilsen NJ, Espevik T, Golenbock DT (2004) TLR9 signals after translocating from the ER to CpG DNA in the lysosome. Nat Immunol 5:190-198

Lazarus R, Klimecki WT, Raby BA, Vercelli D, Palmer LJ, Kwiatkowski DJ, Silverman EK, Martinez F, Weiss ST (2003) Single-nucleotide polymorphisms in the Toll-like receptor 9 gene (TLR9): frequencies, pairwise linkage disequilibrium, and haplotypes in three U.S. ethnic groups and exploratory casecontrol disease association studies. Genomics 81:85-91
Malaney P, Spielman A, Sachs J (2004) The malaria gap. Am J Trop Med Hyg 71:141-146

Nino-Vasquez JJ, Vogel D, Rodriguez R, Moreno A, Patarroyo ME, Pluschke G, Daubenberger CA (2000) Sequence and diversity of DRB genes of Aotus nancymaae, a primate model for human malaria parasites. Immunogenetics 51:219-230

Pichyangkul S, YongvanitchitK, Kum-arb U, Hemmi H, Akira S, Krieg AM, Heppner DG, Stewart VA, Hasegawa H, Looareesuwan S, Shanks GD, Miller RS (2004) Malaria blood stage parasites activate human plasmacytoid dendritic cells and murine dendritic cells through a Toll-like receptor 9-dependent pathway. J Immunol 172:4926-4933

Richie TL, Saul A (2002) Progress and challenges for malaria vaccines. Nature 415:694-701

Rutz M, Metzger J, Gellert T, Luppa P, Lipford GB, Wagner H, Bauer S (2004) Toll-like receptor 9 binds single-stranded CpG-DNA in a sequence- and pH-dependent manner. Eur J Immunol 34: $2541-2550$

Saitou N, Nei M (1987) The neighbor-joining method: a new method for reconstructing phylogenetic trees. Mol Biol Evol 4:406-425

Vecino W, Daubenberger C, Rodriguez R, Moreno A, Patarroyo M, Pluschke G (1999) Sequence and diversity of T-cell receptor beta-chain $\mathrm{V}$ and $\mathrm{J}$ genes of the owl monkey Aotus nancymaae. Immunogenetics 49:792-799

Vollmer J, Weeratna R, Payette P, Jurk M, Schetter C, Laucht M, Wader T, Tluk S, Liu M, Davis HL, Krieg AM (2004) Characterization of three $\mathrm{CpG}$ oligodeoxynucleotide classes with distinct immunostimulatory activities. Eur J Immunol 34:251-262 